DRAFT VERSION OCTOBER 12, 2018

Preprint typeset using LTEX style emulateapj v. 5/2/11

\title{
THE PROPERTIES OF HYPERVELOCITY STARS AND S-STARS ORIGINATING FROM AN ECCENTRIC DISC AROUND A SUPERMASSIVE BLACK HOLE
}

\author{
LADISLAV ŠUBR ${ }^{1}$ AND JAROSLAV HAAS ${ }^{1}$ \\ ${ }^{1}$ Charles University in Prague, Faculty of Mathematics and Physics, Astronomical Institute, V Holešovičkách 2, Praha, CZ-18000, Czech Republic \\ Draft version October 12, 2018
}

\begin{abstract}
Hypervelocity stars (HVSs) that are observed in the Galactic halo, are believed to be accelerated to large velocities by a process of tidal disruption of binary stars passing close to a supermassive black hole (SMBH) which resides in the center of the Galaxy. It is, however, still unclear, where these relatively young stars were born and which dynamical process pushed them to nearly radial orbits around the SMBH. In this paper we investigate the possibility that the young binaries originated from a thin eccentric disc, similar to the one observed in the Galactic center nowadays. By means of direct $N$-body simulations, we follow the dynamical evolution of an initially thin and eccentric disc of stars with a 100\% binary fraction orbiting around the SMBH. Such a configuration leads to Kozai-Lidov oscillations of orbital elements, bringing considerable amount of binaries to close vicinity of the black hole. Subsequent tidal disruption of these binaries accelerates one of their component to velocities well above the escape velocity from the SMBH while the second component becomes tightly bound to the SMBH. We describe the main kinematic properties of the escaping and tightly bound stars within our model and compare them qualitatively to the properties of the observed HVSs and S-stars, respectively. The most prominent feature is a strong anisotropy in the directions of the escaping stars which is observed for the Galactic HVSs but not explained yet.

Subject headings: Galaxy: halo - Galaxy: nucleus - black hole physics - methods: numerical - stars: kinematics and dynamics - stars: early-type
\end{abstract}

\section{INTRODUCTION}

Hypervelocity stars (HVSs) are observed in the Galactic halo at distances $\gtrsim 50 \mathrm{kpc}$ from the Galactic center, moving at high velocities $\gtrsim 300 \mathrm{~km} \mathrm{~s}^{-1}$, i.e. they are not gravitationally bound to the Galaxy. To date, about twenty of such stars were found (Brown et al. 2014), all having spectral type B. The particular location and spectral type is, however, just an observational selection. First, in the Galactic halo, the number density of indigenous stars is low, making the survey of HVSs efficient. Second, the B-type stars are luminous enough to represent convenient targets at such large distances. At the same time, these stars are long lived enough to be able to travel from the Galaxy to the halo. It is likely that near future observations will reveal a number of less luminous HVSs (see Vickers et al. 2015 for one of the first lists of low mass HVS candidates).

S-stars lie, in a certain sense, on the opposite side of the fiducial Galactic scale from the HVSs - they are the most tightly bound stars to the supermassive black hole (SMBH) residing in the center of the Galaxy (e.g. Ghez et al. 2005; Gillessen et al. 2009; (Meyer et al. 2012). Semi-major axes of their orbits range from $\approx 0.004 \mathrm{pc}$ to $\approx 0.04 \mathrm{pc}$. Similarly to the HVSs, the S-stars are classified as main sequence B type stars and, similarly to the HVSs, there are observational limitations which do not allow to detect less luminous main sequence stars in that region.

It was suggested by Hills (1988) already before finding any HVSs or S-stars that both groups (considering their kinematic state, not necessarily the spectral type) should exist as a consequence of tidal break-ups of binary stars in the vicinity of the SMBH. Subsequent works of other authors, particularly after the discovery of the S-stars and the HVSs, tried to an-

E-mail: subr@sirrah.troja.mff.cuni.cz, haas@sirrah.troja.mff.cuni.cz swer the question where these binary stars were born and which process drove them to orbits plunging below the tidal break-up radius. Some works considered the binaries to originate at distances well above $1 \mathrm{pc}$ from the SMBH and to be transported inwards, e.g., within a massive young star cluster (Gould \& Quillen 2003) or scattered due to relaxation enhanced by massive perturbers (Perets et al. 2007).

Another possible source of binaries appears to be quite straightforwardly motivated by presence of another group of young stars in the Galactic center, which are observed at radial distances $0.04 \mathrm{pc} \lesssim r \lesssim 0.4 \mathrm{pc}$ from the $\mathrm{SMBH}$ (e.g. Paumard et al. 2006; Bartko et al. 2009, 2010; Do et al. 2013). A subset of these stars apparently orbit around the SMBH in a thin disc (the so-called clockwise disc, CWS; e.g. Levin \& Beloborodov 2003). Their binary fraction is currently not well constrained (e.g. Pfuhl et al. 2014) either from the observational data, or from models of star formation in the vicinity of the SMBH. In spite of the fact that stars from the young stellar disc have moderate values of orbital eccentricities, their pericenters are several orders of magnitude above the tidal break-up radius of binaries that can survive in such a dense environment. Hence, some mechanism has to come in act to excite the orbital eccentricities in order to transport the binaries sufficiently close to the SMBH.

Löckmann et al. (2008) suggested that the sought mechanism may be the Kozai-Lidov resonance, induced by external, flattened and axially symmetric source of gravity. In their particular setup it was a second, highly inclined stellar disc which was assumed to be formed at the same time in the Galactic center. Later on, Madigan et al. (2009) who studied dynamics of an eccentric disc of stars around a SMBH noticed that substantial fraction of stellar orbits undergo extreme oscillations of their eccentricities due to the disc self-gravity. Madigan et al. (2009) interpreted these oscillations as a manifestation of the so-called eccentric instability and claimed that 
their occurrence relies on presence of massive spherical cluster centered on the SMBH. In Haas \& Subr (2016) we have shown, however, that these oscillations may be interpreted as an imprint of Kozai-Lidov dynamics in the perturbing gravitational field of the stellar disc itself which does not require presence of the spherical cluster. $\square$

Both Löckmann et al. (2008) and Madigan et al. (2009) suggested that the oscillations of orbital eccentricities which they observed in their models could contribute to formation of HVSs and the S-stars. Note, however, that in both works, only orbits of single stars were integrated in the full $N$-body setup. Conclusions about the HVSs and the S-stars formed through the Hills mechanism were based on numbers of single stars reaching such a vicinity of the SMBH, where a binary would tidally break up. Another class of numerical models of the HVSs and the S-stars is based on integrations of individual binaries injected towards the SMBH with arbitrarily given impact parameters (e.g. Kenyon et al. 2008; Antonini et al. 2010; Zhang et al. 2013). Such experiments allow to evaluate statistical properties of the captured and ejected stars, however, the weak point of the models lies in poorly constrained input parameters of the orbits around the SMBH prior the tidal break-up.

In this paper, we make another step forward by including stellar binaries into $N$-body models of stellar discs around a $\mathrm{SMBH}$. For the first time, this setup allows us to make predictions about properties of the HVSs and the S-stars originating for a young stellar disc in a self-consistent way. Similarly to Madigan et al. (2009) and Haas \& Subr (2016) we consider the parent disc of the young stars to be formed by aligned eccentric orbits, i.e. the disc itself is capable to push some of its members to extremely oscillating orbits.

\subsection{The Kozai-Lidov mechanism}

The key process which we assume to drive stellar orbits to highly eccentric state is the Kozai-Lidov resonance. This resonance belongs to a class of nearly Keplerian motions around a dominating central mass which is perturbed by some source of gravity. In the case of the classical Kozai-Lidov mechanism (Kozai 1962; Lidov 1962) the perturbing gravitational potential is assumed to be axially symmetric, which implies a conservation of the projection of the angular momentum of the orbiting body onto the symmetry axis (also called the Kozai-Lidov integral). As the remaining components of the angular momentum vector may change, it is possible for the orbit to undergo oscillations from highly eccentric with a low inclination with respect to the plane of the perturbation to low eccentric and highly inclined. The classical Kozai-Lidov oscillations were considered by Löckmann et al. (2008) who assumed two mutually highly inclined discs to influence each other.

When the axial symmetry of the perturbing potential is lost, none of the components of the angular momentum is an integral of motion any longer. A specific class of sources of such a perturbing potential relevant for celestial mechanics - body on an elliptical orbit around the central mass - was studied in the literature (e.g. Ford et al.2000; Katz et al.2011; Lithwick \& NaOz 2011; Li et al. 2014). If the deviation from the axial symmetry is not strong, the component of the angular momentum vector perpendicular to the plane of the orbit of

\footnotetext{
${ }^{1}$ For further discussion of the eccentric instability see J. Haas \& L. Šubr (2016), in preparation.
}

the perturbing body slowly changes, which leads to modulations of the classical Kozai-Lidov oscillations. The eccentricity of the perturbing body motivates us, as well as the previous authors, to call this effect the eccentric Kozai-Lidov mechanism. In our specific setup with similar orientations of stellar orbits, the most important variant is then the coplanar eccentric Kozai-Lidov mechanism (Li et al. 2014).

\section{MODEL AND METHOD}

\subsection{Model of the Galactic center}

In order to study the role of the Kozai-Lidov mechanism for production of the HVSs and the S-stars, we introduce a model of mutually gravitationally interacting point masses (particles). As there is no explicit characteristic physical scale in the equations of motion of point masses interacting according to the Newton's law of gravity, the model may be arbitrarily rescaled provided the relation $t_{\mathrm{u}}^{2}=r_{\mathrm{u}}^{3} / G M_{\mathrm{u}}$ is fulfilled, where $t_{\mathrm{u}}, r_{\mathrm{u}}$ and $M_{\mathrm{u}}$ represent the time, length and mass unit, respectively, and $G$ stands for the gravitational constant. While in the numerical realization it is convenient to set $t_{\mathrm{u}}=r_{\mathrm{u}}=G M_{\mathrm{u}}=1$, below we describe our model scaled to physical units to make it human readable. In particular, we consider values motivated by the Galactic center: $M_{\mathrm{u}}=4 \times 10^{6} M_{\odot}$ and $r_{\mathrm{u}}=0.004 \mathrm{pc}$, which implies $t_{\mathrm{u}}=1.89 \mathrm{yr}$. We integrated numerically two different initial settings, labeled $A$ and $B$ :

(i) $\mathrm{SMBH}$ particle of mass $M_{\bullet}=1 M_{\mathrm{u}}=4 \times 10^{6} M_{\odot}$ is initially at rest at the origin of the coordinate system

(ii, A) 2000 stars are drawn randomly from a power-law distribution function $n_{M_{\star}} \propto M_{\star}^{-1.5}$ with a lower and an upper boundary $1 M_{\odot}$ and $150 M_{\odot}$, respectively, which implies the total mass of the disc $M_{\mathrm{d}} \approx$ $24500 M_{\odot}$. This distribution function is motivated by a recent analysis of the properties of the young stars in the Galactic center done by Lu et al. (2013).

(ii, B) 2000 stars have equal mass of $M_{\star}=12.25 M_{\odot}$, i.e. $M_{\mathrm{d}}=24500 M_{\odot}$ as in model A

(iii, A) All stars are paired to binaries with a preference for the mass ratio close to unity. This is motivated by the fact, that massive stars in the Galactic field tend to have massive companions. Although this is not true for low-mass stars, we used the close to equalmass pairing for the sake of simplicity. Binary semimajor axes were drawn from the Öpik distribution function (Kobulnicky \& Fryer 2007), $n_{a_{\mathrm{b}}} \propto a_{\mathrm{b}}^{-1}$ with $a_{\mathrm{b}} \in\langle 0.1 \mathrm{AU}, 100 \mathrm{AU}\rangle$; their initial eccentricity was set to zero and their orbital angular momentum parallel to the angular momentum of the disc.

(iii, B) All stars are paired to binaries with initial semi-major axis $a_{\mathrm{b}}=10 \mathrm{AU}$, zero eccentricity and the orbital angular momentum parallel to the angular momentum of the disc.

(iv) Binaries are placed on elliptic orbits around the $\mathrm{SMBH}$ with semi-major axes following a power-law distribution function $n_{a} \propto a^{-1}, \quad a_{\text {in }} \equiv 0.04 \mathrm{pc} \leq$ $a \leq a_{\text {out }} \equiv 0.4$ pc. Inclinations with respect to some reference plane were drawn from a distribution function $n_{i} \propto \sin i, i \in\left\langle 0,2.5^{\circ}\right\rangle$. Motivated by the work of Mapelli et al. (2012), who modeled star formation in the vicinity of the SMBH from infalling gas cloud, we introduced a non-zero radial gradient of eccentricities, namely, we initialized them according to formula 
$e=0.9\left(a-a_{\text {in }}\right) /\left(a_{\text {out }}-a_{\text {in }}\right)$. Furthermore, in tune with their results, we constructed the orbits around the SMBH such that their eccentricity vectors point to a common direction, i.e. the ellipses were mutually aligned, not randomly oriented.

If not specified explicitly otherwise, the results presented below correspond to model $A$.

Let us note here that the models presented above do not involve another important component of the Galactic center - the spherical cluster of old stars. This is due to numerical complications (stability and CPU time consumption) which would be introduced by adding this cluster into the full $\mathrm{N}$ body setup. By means of additional simple models without binaries, however, we discuss the impact of the spherical cluster on the results from the main models in Section 4.

\subsection{Numerical integrator}

We used NBODY6 code (Aarseth 2003) for the numerical integration of the equations of motion. We added to the original code routines for logging of beginnings and endings of regularizations into a binary file. We further introduced an identification index for the SMBH particle and stored the minimum distance of all other particles to the one representing the SMBH reached during the integration. Finally, we altered the decision making algorithm for adding particles to neighbor lists. In particular, we weight the standard distance criterion by the mass of the given particle so that the more massive particles are added to the list even when they are at larger distances than the lighter ones. The most prominent target was the SMBH particle which was, due to this modification, a member of the neighbor lists of all other (star) particles. The modification of the neighbor list influences the integration and increases its stability. Among many runtime options of the NBODY6 code, let us specifically mention that we switched off the internal evolution of the stars as well as the post-Newtonian corrections to the stellar dynamics.

\subsection{Escapers and hypervelocity stars}

Analysis of the escaping stars in our models was done as follows. First, we identified all stars that reached a distance of 8 pc from the SMBH with a velocity exceeding the escape velocity from the SMBH at that radius $\left(\approx 66 \mathrm{~km} \mathrm{~s}^{-1}\right)$. For each of these stars we search the list of the regularization events for the last one including its ID. This event provided us with information about the ejection time, $t_{\mathrm{ej}}$, and radius, $r_{\mathrm{ej}}$. Finally, we scan the event list file for all events in time interval $\left\langle t_{\mathrm{ej}}-\Delta t, t_{\mathrm{ej}}\right\rangle$ involving the respective star. We count the number of unique stellar IDs that occur in these events in order to determine the type of ejection mechanism. The time interval $\Delta t$ is taken to be the greater of the orbital period around the $\mathrm{SMBH}$ with semi-major axis equal to $r_{\mathrm{ej}}$ and $1 t_{\mathrm{u}}$. We explicitly distinguish the ejection process involving just two stars a tidal disruption of binary - which we call the Hills mechanism throughout the rest of the paper. The other ejection cases that involve more than two stars are called multi-body ejections. The method of determination of the ejection event as well as the number of stars involved depend on the details of the regularization techniques implemented in the NBODY6 code which are not designed directly for this purpose. Therefore, there is a small fraction (of the order of one per cent) of the escaping stars whose $r_{\mathrm{ej}}, t_{\mathrm{ej}}$ and mechanism of the ejection may be misdetected.
Not all of the stars which reach the distance of $8 \mathrm{pc}$ with velocity larger than the escape velocity from the SMBH would also reach the Galactic halo. In order to present results suitable for a comparison with the observed HVSs, we performed a reduction of velocities which accounts for the loss of the kinetic energy in the potential of the Galaxy. In particular, we considered a relatively simple compound spherical potential of the Galaxy and the SMBH (e.g. Kenyon et al. 2008),

$$
\begin{aligned}
\Phi(r) & =\Phi_{\mathrm{G}}(r)+\Phi_{\bullet}(r) \\
& =2 \pi G C r_{\mathrm{c}}^{2}\left[\frac{2 r_{\mathrm{c}}}{r} \arctan \frac{r}{r_{\mathrm{c}}}+\ln \left(1+\frac{r^{2}}{r_{\mathrm{c}}^{2}}\right)\right]-\frac{G M_{\bullet}}{r},
\end{aligned}
$$

where $C$ and $r_{\mathrm{c}}$ are free parameters of the model for which we adopt values $C=1.4 \times 10^{4} M_{\odot} \mathrm{pc}^{-3}$ and $r_{\mathrm{c}}=8 \mathrm{pc}$. Specific kinetic energy of stars then decreases by a value of $\Delta E_{\mathrm{K}} \approx 4 \times 10^{5}\left(\mathrm{~km} \mathrm{~s}^{-1}\right)^{2}$ when traveling from $8 \mathrm{pc}$ to $50 \mathrm{kpc}$, i.e. from the radius where the Galactic potential starts to dominate to the typical Galactocentric distance of the observed HVSs. In the following, we thus distinguish all the stars escaping from the $S M B H$ at the distance of $8 \mathrm{pc}$ (or shortly escapers) and the hypervelocity stars (HVSs) which are the subset of escapers capable to reach the galactocentric distance of $50 \mathrm{kpc}$ (i.e. having velocities greater than $894 \mathrm{~km} \mathrm{~s}^{-1}$ at $\left.8 \mathrm{pc}\right)$.

\subsection{S-stars}

Formally, we define S-stars in our model to be the former companions of the escaping stars originating from the Hills mechanism which stay bound to the SMBH. We will show below that an overwhelming majority of these stars are located below the inner edge of the parent stellar disc, i.e. at the places where the observed S-stars are found in the Galactic center. Orbital elements of the S-stars are evaluated in the rest frame of the $\mathrm{SMBH}$ particle.

\section{RESULTS}

\subsection{Properties of the hypervelocity stars}

We have integrated 150 realizations of model $A$ up to $t=4 \times 10^{6} t_{\mathrm{u}}$ which corresponds to $t \approx 7.6 \mathrm{Myr}$ for scaling $r_{\mathrm{u}}=0.004 \mathrm{pc}$ and $M_{\mathrm{u}}=4 \times 10^{6} M_{\odot}$. On average, we found 34 escaping stars per realization out of which approximately $5 / 6$ originated from the Hills mechanism while the remaining $1 / 6$ accounts for other modes of ejection - either a strong interaction of a single star with a binary, or the binary-binary scattering. In some rare cases, the event list does not contain any information about regularized interactions with other stars, which may in reality correspond to any of the above mentioned mechanisms or even a close hyperbolic interaction of two single stars in the tidal field of the SMBH. We excluded these stars from further considerations. After the velocity correction to the Galactic potential, we obtain on average $\approx 12$ HVSs per realization.

Figure 1 shows the distributions of the absolute values of the velocities of all escaping stars at $8 \mathrm{pc}$ and of their subset ejected via the Hills mechanism within model $A$ (thick and thin solid lines, respectively). Comparison of the two distributions clearly shows that the Hills mechanism dominates the ejection process for $v \gtrsim 500 \mathrm{~km} \mathrm{~s}^{-1}$ at $r=8 \mathrm{pc}$. Consequently, the potentially observable HVSs at $r=50 \mathrm{kpc}$ practically exclusively originate from the Hills mechanism. Dashed lines in Figure 1 represent expected velocity distributions of the HVSs at $r=50 \mathrm{kpc}$, i.e. distributions of veloc- 


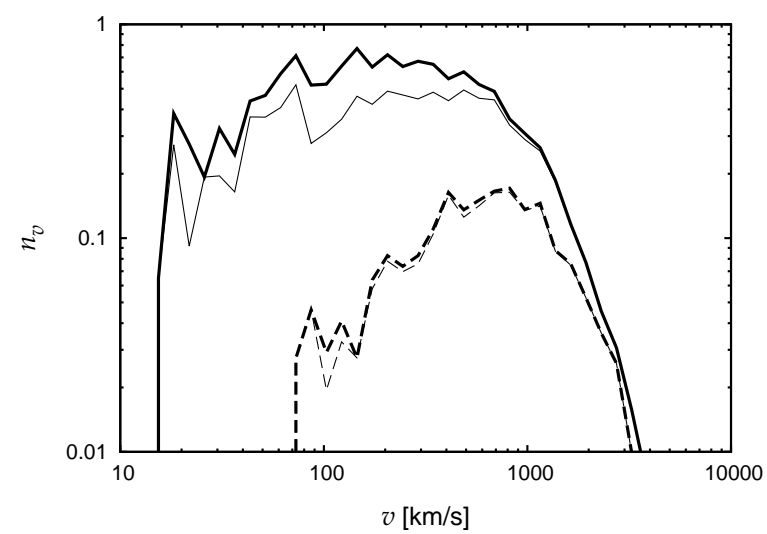

FIG. 1.- Arbitrarily normalized velocity distributions of escaping stars detected at a distance of $8 \mathrm{pc}$ from the SMBH within model A (solid lines). Dashed lines represent the velocity distributions of the HVSs that would be reached at a distance of $50 \mathrm{kpc}$ from the Galactic center. Thick lines show velocity distributions of all escapers, while the thin ones correspond to the stars ejected via the Hills mechanism.

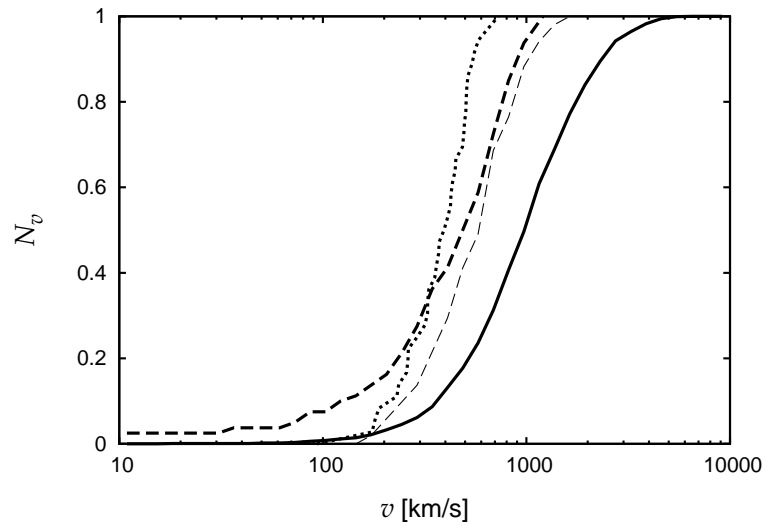

FIG. 2.- Cumulative distribution function of velocities of HVSs at $r=$ $50 \mathrm{kpc}$ from models A and B are plotted with solid and dashed lines, respectively. Thin dashed line shows how the distribution function changes when deeper gravitational potential well of the Galaxy is assumed. Dotted line represents distribution function of Galactic rest-frame velocities of HVSs reported by Brown et al. (2014), including those declared as potentially bound to the Galaxy. The velocities are reduced to the same galactocentric distance of $50 \mathrm{kpc}$ under the assumption that they move in the potential described by formula (1).

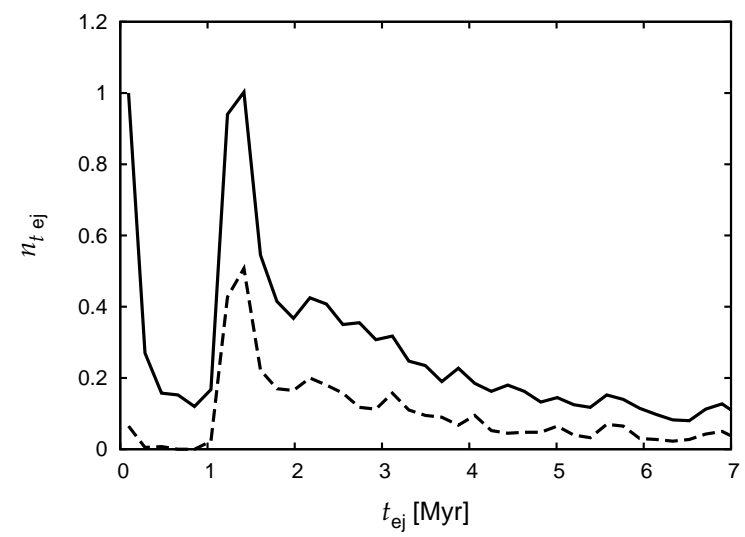

FIG. 3.- Arbitrarily normalized distribution functions of the ejection times of all escaping stars in model $\mathrm{A}$ at $r=8 \mathrm{pc}$ (solid lines) and the HVSs (dashed lines). ities of the HVSs reduced by motion in the Galactic potential (1).

Model A appears to give velocity distribution considerably broader and shifted to higher velocities with respect to the observed HVSs (see Figure 2). Figure 2, however, also shows that the velocity distribution of the HVSs strongly depends on the initial properties of the stellar disc. Model B which starts with all binaries initially relatively weakly bound $\left(a_{\mathrm{b}}=10 \mathrm{AU}\right)$ gives considerably less HVSs with velocities exceeding $1000 \mathrm{~km} \mathrm{~s}^{-1}$ at $50 \mathrm{kpc}$. This can be quite naturally explained by the fact that the more tightly bound binaries from model $\mathrm{A}$ get tidally disrupted closer to the $\mathrm{SMBH}$, passing it with larger velocity which is then inherited by the escaping star. Let us further note that distribution function of binary semi-major axes in model B evolved (broadened) quite rapidly due to two-body relaxation. Typical semi-major axis of tidally broken-up binaries which led to HVSs in model B was about $1 \mathrm{AU}$.

Figure 2 also demonstrates that the distribution of velocities of the HVSs is sensitive upon the shape of the Galactic potential. The two distributions plotted for model B (dashed lines) differ by the amount of reduced kinetic energy - the thick line corresponds to $\Delta E_{\mathrm{K}}=4 \times 10^{5}\left(\mathrm{kms}^{-1}\right)^{2}$, according to formula (1), while the thin line corresponds to $\Delta E_{\mathrm{K}}=5 \times 10^{5}\left(\mathrm{~km} \mathrm{~s}^{-1}\right)^{2}$. Note that the simple model of Galactic potential (1) may have similar level of inaccuracy. For example, while its parameters are such that it reasonably well fits mass of the Galactic nuclear star cluster within the distance of $\approx 8 \mathrm{pc}$ from the SMBH, it very likely does not reproduce well the density distribution in that region and, consequently, it underestimates depth of the Galactic potential. The way how reduction of kinetic energy translates to change of velocities directly implies that stars with lower velocities are affected more as it can be also inferred from Figure 2

Considering large uncertainty in initial properties of the stellar disc together with uncertainty of the depth of the Galactic potential, we are not able to provide unique prediction on the velocity spectrum of the HVSs which may be produced by the mechanism described in this paper. Some level of uncertainty has to be also considered on the observational side when comparing models to the observational data.

In Figure 3, we plot the distribution function of the ejection times of the escapers within model A. Again, we distinguish distributions of all escapers and the HVSs. We see an initial peak at $t=0$ which is especially apparent for the overall distribution, i.e. it is dominated by the low velocity escapers. These originate from those primordial binaries that are immediately broken up in the tidal field of the SMBH. More interesting is the relatively narrow peak of ejection of the HVSs which rises at $t \approx 1 \mathrm{Myr}$ and slowly decays during the subsequent few million years. This feature is a consequence of the coplanar eccentric Kozai-Lidov mechanism, which is responsible for the production of the majority of the HVSs in our setup. Our results show that most of the oscillating orbits are located close to the inner edge of the disc, thus having similar characteristic time-scale of the eccentric Kozai-Lidov cycles. Hence, a large fraction of the affected binaries reach high eccentricity at a common time. Typical representative of the tidally disrupted binary is shown in Figure 4. Initially, the lines represent orbital elements of the binary barycenter. At $t=1.15 \mathrm{Myr}$, eccentricity of its orbit around the $\mathrm{SMBH}$ reaches value $e>0.99$ and the binary breaks up. From that time on, the lines in Figure 4 repre- 


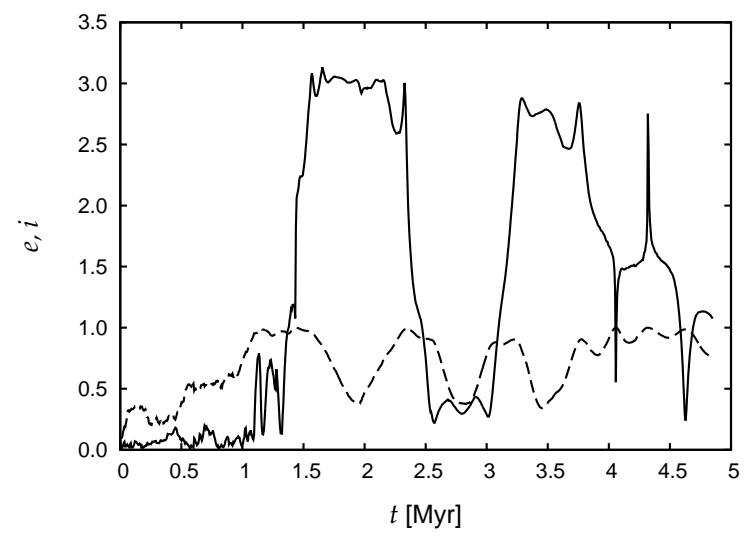

FIG. 4.- Temporal evolution of inclination (solid line) and eccentricity (dashed) of an orbit from the inner part of the disc. Tidal break-up occurs at $t=1.15 \mathrm{Myr}$. From that time on, orbital elements of the component that remains bound to the SMBH are plotted.

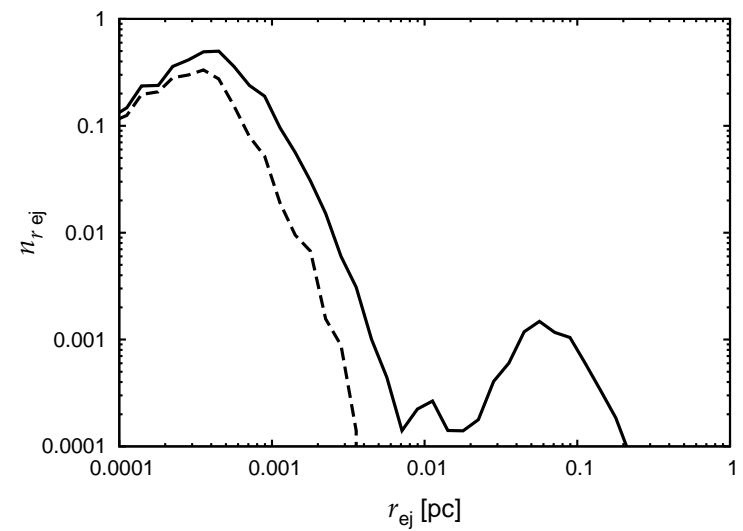

FIG. 5.- Arbitrarily normalized distribution functions of the ejection radii of all escaping stars in model A at $r=8 \mathrm{pc}$ (solid line) and the HVSs (dashed line).

sent orbital elements of the component which remains bound to the SMBH, while the other one escapes. Oscillations of orbital elements of the bound star shows pattern typical for the coplanar eccentric Kozai-Lidov cycles (Li et al. 2014; see also Haas \& Subr 2016) with flips from corotation to counterrotation and vice versa. As the time proceeds, pattern of the oscillations changes which is likely due to evolution of the source of the perturbing potential (the stellar disc) in the $N$-body environment. Semi-major axis of the bound star just after the tidal break-up is $a \approx 0.015 \mathrm{pc}$ which is approximately one half of that of the binary before that event. The escaping star becomes unbound to the $\mathrm{SMBH}$, having velocity of $\approx 140 \mathrm{~km} \mathrm{~s}^{-1}$ at a distance of $8 \mathrm{pc}$ from the SMBH, i.e. this particular star does not contribute to the population of the HVSs.

Figure 5 demonstrates that tidal break-ups of binaries within model $A$ occur in a relatively wide range of radii. This is caused by an initially wide range of the (intrinsic) binary semi-major axes considered in our model which determine how deep into the potential well of the SMBH the binary can penetrate before being tidally broken up. We also see that HVSs practically exclusively originate from radii $\lesssim 0.0005$ pc.

Figure 6 shows positions of the escaping stars on the sphere

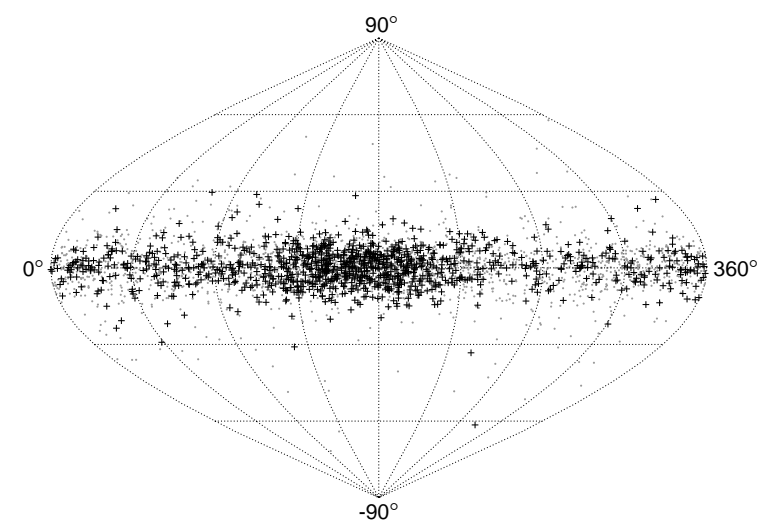

FIG. 6.- Distribution of the position vectors of the escaping stars projected in model $\mathrm{A}$ on a sphere centered on the SMBH; longitude is measured in the plane of the stellar disc; latitude is the angle measured from the stellar disc plane. Crosses represent HVSs, while the gray dots stand for the rest of the escaping stars, i.e. those which would not reach galactocentric distances $\gtrsim 50 \mathrm{kpc}$.

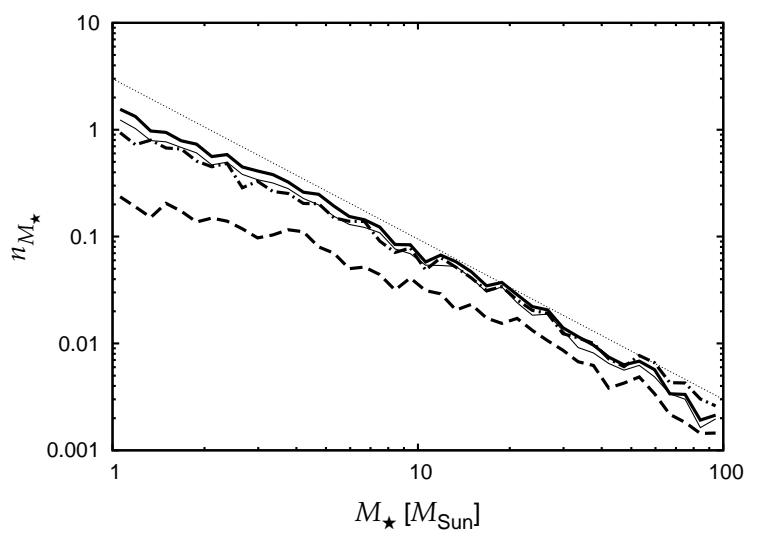

FIG. 7.- Mass functions of different groups of stars in model A: all escaping stars (thick solid line), all escaping stars ejected via the Hills mechanism (thin solid), HVSs (thick dashed) and the S-stars (thick dash-dotted). Thin dotted line represents the initial mass function of the disc.

in the sinusoidal projection. There is a clear anisotropy in the distribution with majority of stars escaping along the plane of the stellar disc. This is a natural consequence of the coplanar eccentric Kozai-Lidov mechanism which is the main process that brings the binaries from the disc to extremely eccentric orbits in our models. In spite of the fact that the orbits change their inclination during the secular evolution, this only happens hand-in-hand with evolution of eccentricities. In particular, in the high-inclination phase $\left(i \approx 90^{\circ}\right)$, the eccentricity is so large and argument of pericenter is close to 0 or $\pi$ that the orbit is embedded in the disc and the velocity vector of the star is nearly parallel with the disc plane for major part of the orbit. It is then natural to expect that the unbound component of a tidally broken up binary follows a trajectory nearly parallel to the stellar disc. Beside the strong tendency of escaping at low latitudes with respect to the disc, we also observe a significant clustering of the ejected stars around a certain value of the azimuthal angle in the plane of the disc. We attribute this to the nature of the eccentric Kozai-Lidov mechanism which relies on specific mutual orientation of the orbit and the eccentric perturbation of the central potential. In spite of that the current observations are incomplete in sky-coverage, they 

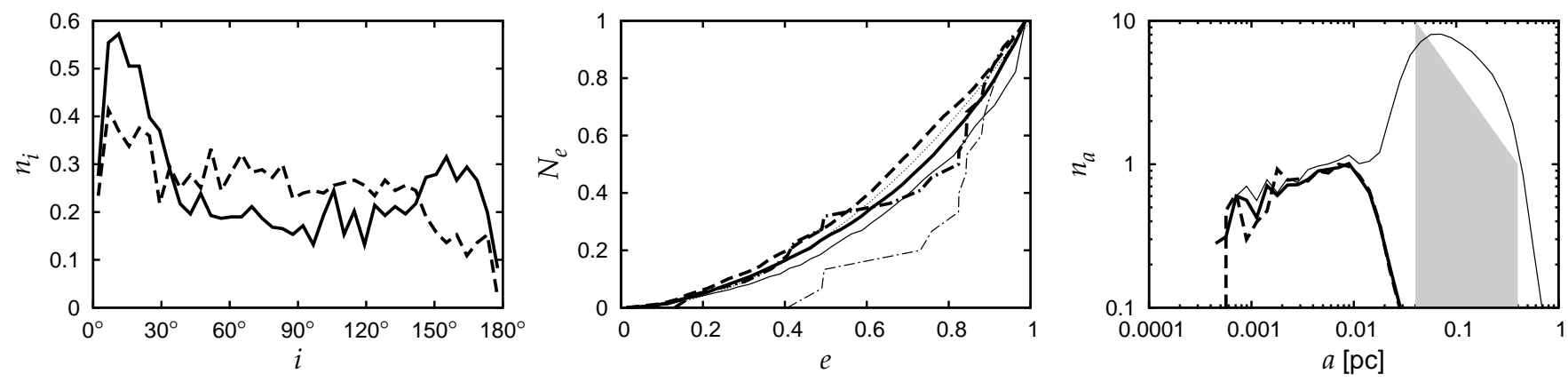

FIG. 8.- Arbitrarily normalized distribution functions of inclinations and semi-major axes and a cumulative distribution function of eccentricities of the S-stars according to the model $A$. Thick solid lines show the distributions of the S-stars produced within the first $3.8 \mathrm{Myr}$ and they also represent the initial state of the follow-up model. Thick dashed lines describe the final state of the follow-up integration. Thin dotted line in the middle panel corresponds to thermal eccentricity distribution, $N_{e} \propto e^{2}$, thick and thin dash-dotted lines show eccentricity distribution of observed young stars in the Galactic center and of their subset with $a<1^{\prime}$, respectively. Thin solid line in the middle panel depicts eccentricity distribution within the model B. Thin solid line in the right panel shows distribution function of semi-major axes of all stars within model $\mathrm{A}$ at $t=3.8 \mathrm{Myr}$, while the shaded area represents its initial state.

indicate statistically significant anisotropy of distribution of the HVSs on the sky (Brown et al.2014), which is in a qualitative accord with the outcome of our model.

The currently observed HVSs are exclusively late B-type stars (e.g. Brown et al. 2014), which is, nevertheless, just a selection effect and the mass spectrum of the HVSs has to be considered unknown at this time. The numerical model, however, enables us to predict the mass spectrum of the HVSs. Figure 7 shows the mass function of the escaping stars produced during the whole integration of model $A$. We see that it somewhat deviates from the initial mass function, in particular, it is flatter at the low-mass end. This feature is more prominent for the HVSs, i.e. it is primarily these stars that are responsible for the deviation from the initial mass function. We see two possible reasons for such a flattening of the mass function which are not mutually exclusive. First, more massive binaries have on average a larger binding energy than the lighter ones, i.e. they are able to sink deeper into the potential well of the SMBH before tidally breaking up. Consequently, they achieve higher ejection velocities, i.e. they have larger probability to be detected as HVSs. Second, and a probably more important reason is that it is preferably the more massive stars (binaries) which sink towards the SMBH within the parent disc due to two-body relaxation. In spite of the fact that this process is not as pronounced as in self-gravitating systems without a dominating central mass, our simulations show that the mass function at the inner edge of the disc gets somewhat flatter already at $t \approx 1 \mathrm{Myr}$. As the eccentric Kozai-Lidov cycles have a shorter period at smaller radii in our settings, they preferentially push binaries from this region to highly eccentric orbits on which they can tidally break up.

\subsection{S-stars}

Let us now analyze the properties of the S-stars in our model as they were defined in Section 2.4. On average we obtain $\approx 28 \mathrm{~S}$-stars per realization of model $\mathrm{A}$ up to $t=7.6 \mathrm{Myr}$ In the subsequent paragraphs, we will also qualitatively discuss comparison of the properties of the Sstars in our model with those of the observed in the Galactic center. Note, however, that such a comparison has to be handled with caution for two main reasons. First, the number of the observed S-stars is quite small $(\approx 20)$ which makes any statistical analysis only marginally reliable. Second, we have

\footnotetext{
${ }^{2}$ In $\approx 5 \%$ of the binary tidal break-ups, both components were accelerated above the escape velocity from the SMBH.
}

strict definition of S-stars in this paper based on their dynamical history. Such a definition cannot be applied to the stars observed in the Galactic center. Actually, to our knowledge, there is not any widely accepted definition of the observed Sstars. Therefore, it is very difficult to compare the outcomes of numerical models with the observational data.

Figure 8 shows main statistical properties of the captured S-stars. Left panel presents the distribution of their inclinations within model $A$. We see two dominant peaks around $i=0$ and $\pi$, i.e. corotating and counterrotating with respect to the parent disc. Similarly to the distribution of the velocity vectors of the ejected stars, the high anisotropy of the orientations of the orbits of the S-stars is a consequence of the Kozai-Lidov mechanism. Despite the vague definition of the observed S-stars, it is quite safe to state that the young stars observed within the projected distance of $\approx 1^{\prime \prime} \approx 0.04 \mathrm{pc}$ from the SMBH have relatively randomly oriented orbital planes (at least in comparison to the coherent orientations of orbits of the stars forming the young stellar disc above this radius). Hence, there has to be some process which randomizes the orbital orientations in order to smear out their high anisotropy introduced by the process of their formation discussed in this paper. Indeed, it was suggested by various authors that the orientations of the orbits of the S-stars may have undergone considerable evolution due to stellar dynamics. In particular, resonant relaxation processes (Rauch \& Tremaine 1996) within the nuclear star cluster were discussed e.g. by Hopman \& Alexander (2006). Another process capable of changing the orientations of the orbits is the Kozai-Lidov oscillations due to a secondary, arbitrarily inclined stellar disc (Löckmann et al. 2008). A certain combination of the two processes was considered by Chen \& Amaro-Seoane (2014). Our model does not allow us to verify the resonant relaxation of the orbital parameters of the S-stars as it does not include the spherical component of the nuclear star cluster for numerical reasons. We tried to overcome this limitation by means of a follow-up model. It consists of the S-stars whose initial kinematic state was taken from the main model $A$ at $t=2 \times 10^{6} t_{\mathrm{u}} \approx 3.8 \mathrm{Myr}$, regardless of the time of their formation. Motivation for this time is twofold. On one hand, at $t \approx 3.8 \mathrm{Myr}$, on average a considerable number of S-stars $(\approx 21)$ are already formed in the main model. On the other hand we wish to test whether the orbits of a substantial fraction of the S-stars can be randomized within a few millions of years which is the estimated life-time of the currently ob- 
served young stellar disc in the Galactic center. The S-stars were embedded in a spherical cluster modeled by 500 stars of equal mass $\left(1 M_{\odot}\right)$ with random orientations of their orbits, thermal distribution of eccentricities and a distribution of the semi-major axes $n_{a} \propto a^{1 / 2}$ in $\langle 0.002 \mathrm{pc}, 0.02 \mathrm{pc}\rangle$, i.e. within the domain of the S-stars. Distributions of the orbital elements of the S-stars after 3.8 Myr of the follow-up dynamical evolution are plotted in Figure 8 with the dashed lines. We observe a substantial evolution of the orientations of the orbits which tend to become randomized. Hence, we may state that our model of production of the S-stars via the Hills mechanism is compatible with the orientations of the observed S-stars.

Distribution of eccentricities of the S-stars in our model A appears to be close to the thermal one, $n_{e} \propto e$ (Figure 8 middle panel) and it does not evolve considerably in time when embedded in the spherical cluster. The distribution is roughly compatible with that of early-type stars with determined orbital elements observed in the Galactic center (thick dash-dotted line, data taken from Gillessen et al. 2009). The situation changes if only stars with semi-major axis smaller than $1^{\prime \prime}$ are considered (thin dash-dotted line in Figure 8). Gillessen et al. (2009) reported a super-thermal eccentricity distribution $\left(n_{e} \propto e^{2.6 \pm 0.9}\right)$ for this subset of stars in the Galactic center, which is steeper than what we obtained within the considered models $A$ and $B$. The apparent differences between the outcomes of our two models suggest, however, that further variations of the initial setup may lead to even more super-thermal distribution of eccentricities. Furthermore, in order to obtain more realistic results, the numerical model would have to include all resonant and relaxational processes for the whole integration time. Finally, the comparison with the observational data needs to account for the problem of definition of the S-stars mentioned above.

Showing the distribution of eccentricities of the captured stars, let us mention that works of other authors (e.g. Perets et al. 2009; Madigan et al. 2011; Antonini \& Merritt 2013) discussing origin of the S-stars often expect their eccentricities coming from the Hills mechanism to be exclusively greater than 0.9 . Our results indicate that this assumption may not be valid in the case when the orbit around the SMBH secularly evolves, i.e. the binary becomes gradually more perturbed when passing closer and closer to the SMBH during subsequent revolutions around it. Let us also remark that Hills (1991) obtained extreme values of eccentricities of captured stars only for highly radial orbits, while larger impact parameters led to mean values of eccentricity considerably smaller than 0.9 .

The S-stars produced by the Hills mechanism in model A occupy a rather wide range of semi-major axes: $0.001 \mathrm{pc} \lesssim$ $a \lesssim 0.01 \mathrm{pc}$ (see the right panel of Figure 8). This is in accord with the observational data which currently provide us with the smallest semi-major axis of $\approx 0.004 \mathrm{pc}$ (S0-102, Meyer et al. 2012) while the outermost S-stars are located close to the inner edge of the young stellar disc $(\approx 0.04 \mathrm{pc})$. Comparison of the semi-major axis distribution function of all stars and that of the S-stars in the right panel of Figure 8 indicates that the region below $0.01 \mathrm{pc}$ is exclusively occupied by the S-stars in our model which justifies their formal definition in Section 2.4. As expected, the distribution of the semi-major axes did not change as their two-body relaxation time is several orders of magnitude longer than the integration time.

Finally, we also evaluated the mass function of the captured S-stars in model A. We found it to be similar to that

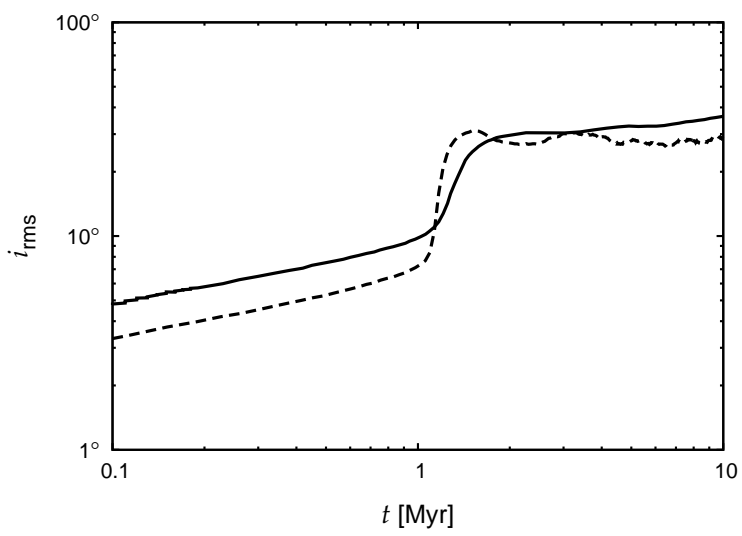

FIG. 9.- Temporal evolution of root mean square inclination of orbits from model A (solid line) and from model M8 of Haas \& Subr (2016; dashed).

of their former companions, i.e. the escaping stars ejected via the Hills mechanism (see Figure 7 and its description in the previous Section). This result is not surprising for the initial setup of our model in which the pairing of stars into binaries was biased towards equal masses.

\subsection{Disc structure evolution}

On time-scale of the order of ten million years, both secular and relaxational processes influence structure of the parent stellar disc. Due to relatively coherent motions of the stars and, therefore, small initial velocity dispersion in the disc, two-body relaxation is capable of altering its radial density profile, making it flatter and broadening its extent both below and above its initial inner and outer edges, respectively (see right panel of Figure 8). Except for the population of S-stars, which were transported below $0.01 \mathrm{pc}$ due to the Hills mechanism, the radial density profile does not differ significantly from that of models consisting of only single stars presented in our previous works (Subr \& Haas 2014; Haas \& Subr 2016).

Figure 9 shows that the two-body relaxation is also mainly responsible for heating up the disc during the initial phase of evolution $(\lesssim 1 \mathrm{Myr})$ which manifests itself by a power-law growth of root-mean-square inclination of the orbits, $i_{\mathrm{rms}} \propto t^{1 / 4}$ (e.g. Stewart \& Ida 2000). At $t \approx 1 \mathrm{Myr}$, accelerated growth of $i_{\mathrm{rms}}$ is observed, which is a consequence of relatively coherent Kozai-Lidov oscillations of a subset of orbits which flip to counterrotation, thus pushing $i_{\text {rms }}$ to high values. The evolution of $i_{\text {rms }}$ is qualitatively the same as that of model M8 from Haas \& Subr (2016) which has the initial global characteristics of the disc (distribution of orbital elements and total mass) identical to the current model $A$. The offset of the two models at $t \lesssim 1 \mathrm{Myr}$ we attribute to faster relaxation of model $A$ which, unlike the former model M8 has a broad mass spectrum. Another additional source of heating of the disc in model $A$ could be the binaries which are not present in model M8. Recent semi-analytical estimates of Mikhaloff \& Perets (2016) show, however, that binaries contribute only marginally to heating of Keplerian stellar discs around SMBHs in comparison to the process of two-body relaxation. Another kind of difference between evolution of $i_{\text {rms }}$ of the two models can be observed at $t \gtrsim 1 \mathrm{Myr}$. Model M8 exhibits damped oscillations of $i_{\text {rms }}$ which is a consequence of longer lasting 


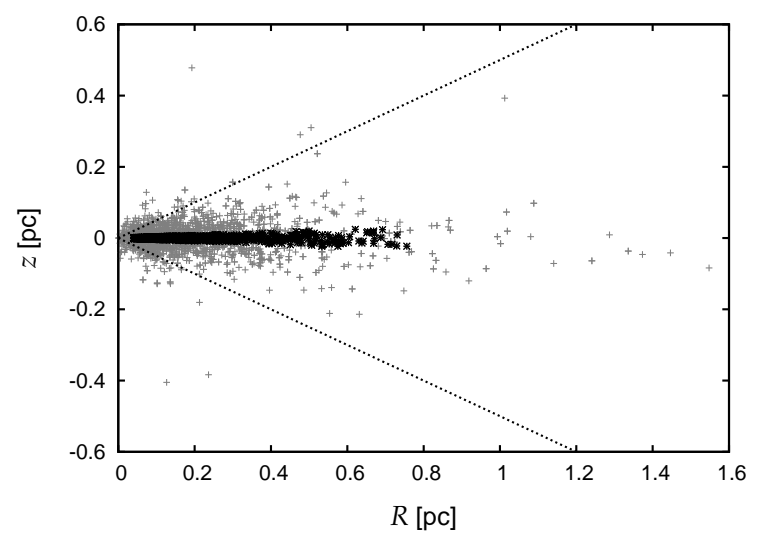

FIG. 10.- Azimuthal projection of position of stars from model $\mathrm{A}$ at $t=$ 7.6 Myr (gray points) overplotted with initial state (black points). Dotted line indicates cone with half-opening angle $30^{\circ}$.

coherence of the Kozai-Lidov oscillations of individual orbits. There are at least two reasons why this feature is not observed in model A. First, tidal disruptions of primordial binaries lead to abrupt changes of orbital elements which affect the Kozai-Lidov cycles. Second, as argued above, model $A$ undergoes faster two-body relaxation due to the mass spectrum which gradually demotes the eccentric perturbation. Both these arguments can also be used to explain higher value of $i_{\mathrm{rms}}$ of model $\mathrm{A}$ at $t \gtrsim 3 \mathrm{Myr}$ which is likely due to orbits that stay frozen in the counterrotating phase after interruption of their Kozai-Lidov cycles.

In Haas \& Subr (2016), we already pointed out that $i_{\text {rms }}$ is not straightforwardly related to the disc geometrical thickness. Argumentation for this statement is basically identical to that in Section 3.1 of the current paper: undergoing the coplanar Kozai-Lidov oscillations, the orbits reach high inclination state with such values of eccentricity and argument of pericentre that they are still embedded in a relatively thin disc structure. Azimuthal projection of a snapshot of one realization of model $A$ at $t=7.6 \mathrm{Myr}$ presented in Figure 10 confirms that except for several outliers, the stellar disc is well confined within a cone with half-opening angle $\approx 30^{\circ}$.

\section{DISCUSSION}

We showed by means of a relatively simple model that both the S-stars and the HVSs may have common origin in an eccentric stellar disc formed by fragmentation of a gaseous cloud falling onto the SMBH. Provided they were born as binaries, growth of the eccentricity of their orbits around the SMBH due to the Kozai-Lidov mechanism induced by the disc itself may have brought them to the tidal break-up radius at which they broke up, leaving one component tightly bound to the SMBH and the other one ejected away at a high velocity. Kinematic properties of the HVSs and the S-stars in our model qualitatively agree with properties of the observed stars. We have to keep in mind, however, that our knowledge of these properties is incomplete due to the observational limits and, therefore, a model matching such incomplete observational data may actually be wrong. In this point, only improved observational data that are supposed to be available in the future may help to test our model. Until then, there is still room for improvements of the model to make it more realistic.

When, e.g., considering the stars as finite-size objects rather than point masses, we may expect them to physically collide. It was suggested by various authors that binary stars orbiting a SMBH may merge due to the secular evolution of their (binary) eccentricity (e.g. Antonini et al. 2010, 2011; Prodan et al. 2015; Stephan et al. 2016). This event may prevent the production of the HVSs provided the binary components merge before they reach the tidal break-up radius. Whether or not the stellar collision occurs depends on a complicated mixture of poorly constrained parameters that determine the time-scales of the binary Kozai-Lidov oscillations and the Kozai-Lidov cycles of the orbit around the SMBH. The fraction of binaries that merge due to oscillations of the binary eccentricities varies from few per cent (Prodan et al. 2015) up to 10\% (Stephan et al. 2016). It is, however, not clear, whether the binaries that produce HVSs in our model would be affected on a similar level. In other words, the fraction of merging binaries is likely to vary across the whole parameter space and we do not know, what is its value in those parts which contribute to production of the HVSs.

Production rate of the HVSs and the S-stars could also change when another sources of gravity capable of modifying the Kozai-Lidov dynamics are involved. One of the observed and theoretically expected constituents of the Galactic nucleus that was neglected in our main models is the old component of the nuclear star cluster (which consists of late-type stars and very likely also compact stellar remnants). Considering it to be spherically symmetric to the first order of approximation, it generally has a tendency to damp the KozaiLidov oscillations. This effect for the case of an axisymmetric perturbation causing these oscillations was discussed, e.g., in Ivanov et al. (2005), Subr et al. (2007) and Karas \& Subr (2007); further extension for the case of an eccentric perturbation, i.e. for the higher order Kozai-Lidov resonances, can be found in Haas \& Subr (2016). In the latter paper, we demonstrated that with an increasing mass of the spherical cluster, the higher order effects of the Kozai-Lidov mechanism are damped first while the classical (quadrupole) cycles can survive larger masses. The limiting mass of the cluster beyond which the Kozai-Lidov mechanism is unable to push the stellar orbits to extreme eccentricities depends on the mass distribution in both the cluster and the disc and also on eccentricities of the stellar orbits. In particular, we found that a moderate to high initial eccentricity of the disc is a key feature as it may lead to a global angular momentum flow through the disc which is capable to push a subset of orbits to higher eccentricities. Consequently, they can reach the resonant configuration which leads to extreme Kozai-Lidov oscillations. Our numerical model in the current paper is not suitable for a direct testing of the influence of the stellar cluster on the production of the HVSs as addition of several tens of thousands of particles would require much more computational time and, at the same time, it would lead to a lower numerical stability. An alternate approach which is commonly used in similar studies, namely, modeling the cluster by a smooth static potential, would require to model the SMBH as an external potential as well, which, however, leads again to a lower numerical stability of our integrations when the binary tidal break-ups occur.

Here we make an attempt to estimate the rate of production of the HVSs and the S-stars based on numerical integrations of models without the primordial binaries, which is a numerically less complicated setup. The key idea lies in the empirical fact, that majority of the tidal break-up events occurred below $0.0005 \mathrm{pc}$ in our model A (see Figure 5). Models with- 


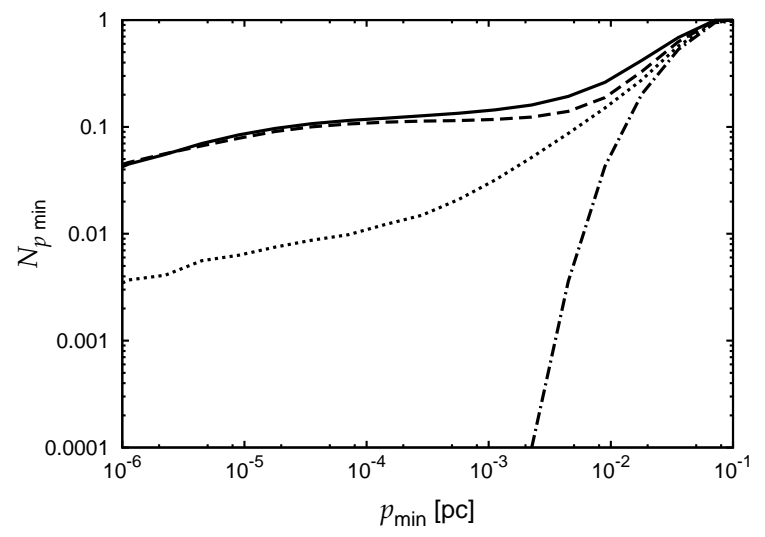

FIG. 11.- Cumulative distributions of the minimal pericentral distance reached in the single-star models with the spherical cluster included. Solid, dashed, dotted and dash-dotted lines correspond to $M_{\mathrm{c}}=0,1 M_{\mathrm{d}}, 4 M_{\mathrm{d}}$ and $10 M_{\mathrm{d}}$, respectively.

out the primordial binaries can then be used to determine the fraction of stars that reach the SMBH to a distance smaller than the given value. Such a fraction can be determined for systems embedded in the spherical potential as well as for the isolated one. In particular, we integrated several models similar to model $A$ introduced in Section 2.1 with the following three key differences:

(i) the $\mathrm{SMBH}$ is modeled as a fixed Keplerian potential

(ii) gravity of the spherical cluster is modeled by a fixed potential $\Phi_{\mathrm{c}} \propto r^{3 / 2}$ which corresponds to a mass distribution $\varrho_{\mathrm{c}}(r) \propto r^{-1 / 2}$. It has a single free parameter, $M_{\mathrm{c}}$, which determines the mass of the cluster enclosed within a radius $a_{\text {out }}$ (the maximum initial value of the semi-major axis of the stellar orbits in the disc).

(iii) all stars are initially single.

We let these models with $M_{\mathrm{c}}=0,1 M_{\mathrm{d}}, 4 M_{\mathrm{d}}$ and $10 M_{\mathrm{d}}$ to evolve for $\approx 10 \mathrm{Myr}$ and monitored the minimal radial distance, $p_{\min }$, of the stars from the $\mathrm{SMBH}$ reached during this time interval. Figure 11 shows the cumulative distribution functions of $p_{\min }$ for the above described models with different values of $M_{\mathrm{d}}$. We see that the spherical cluster of a mass equal to the mass of the disc leads to only small decrease of $N_{p_{\min }}$ at $p_{\min } \approx 0.0005 \mathrm{pc}$ with respect to the isolated system. Therefore, we also do not suppose the number of HVSs to be altered considerably in this case. For $M_{\mathrm{c}}=4 M_{\mathrm{d}}$, the number of stars plunging below $0.0005 \mathrm{pc}$ is lowered by a factor of $\approx 5$ with respect to the case without the spherical cluster included and we expect the number of the HVSs to be lowered by a similar factor, i.e. to $\approx 2$ in contrary to $\approx 12$ obtained for the isolated model (see Section 3.1). Finally, the spherical cluster of mass $M_{\mathrm{c}}=10 M_{\mathrm{d}}$ practically completely damps the Kozai-Lidov oscillations and we do not expect any HVSs or S-stars to be formed via the Hills mechanism within the models described in this paper in such a case (see, however, Haas \& Subr 2016 for discussion of other initial setups in which considerable amount of oscillating orbits occurred even for $M_{\mathrm{c}} \approx 10 M_{\mathrm{d}}$ ). Note that the distribution of $p_{\min }$ does not provide us directly with the expected number of the HVSs as not all particles reaching the critical value would still be in the form of binaries even in the case of a $100 \%$ initial binary fraction and the argumentation above relies on comparison of the isolated models with and without the primordial binaries.
Not only total number of the HVSs and the S-stars formed by the Hills mechanism induced by the Kozai-Lidov oscillations of binary stars orbits around the SMBH could be affected by presence of the spherical stellar cluster. The strong anisotropy of the ejected stars as well as the temporal burst of their production described in Section 3.1 are features of the coplanar eccentric Kozai-Lidov mechanism. Increasing mass of the stellar cluster first damps this mode (Haas \& Subr 2016) and, therefore, we suppose its imprints on the HVSs velocity directions and times of ejection to be suppressed as well. The classical (quadrupole) Kozai-Lidov oscillations are also likely to bring stellar orbits to highly eccentric states with specific orientations of the orbital planes, in this case, nearly parallel to the plane of the disc. Nevertheless, there is no reason to expect preferred direction of the eccentric vector in this plane. In Haas \& Subr (2016) we showed that these classical oscillations occur from the very beginning of the disc evolution till the end of our integrations, thus they are supposed to suppress the dominant peak in the distribution of ejection times of the HVSs. Single-star models introduced above are not suitable for determination of properties of escaping stars. Therefore, complex models including large fraction of primordial binaries in the disc as well as gravity of the spherical cluster deserve to be a subject of future investigations.

There is growing evidence for tidal disruptions of stars in the vicinity of SMBHs in distant galaxies (see e.g. Saxton et al.2012; Komossa 2015). An interesting side-effect of the tidal break-ups of binaries on orbits undergoing the Kozai-Lidov oscillations is that it may prevent the individual stars from being tidally disrupted. The Kozai-Lidov oscillations change the orbital elements of orbits around the SMBH regardless of the multiplicity of the orbiting system. At the maximum of eccentricity, the pericenter may lie below the stellar tidal radius, i.e. if the system were formed by a single star, it would be disrupted. However, a binary tidally breaks up before it reaches the stellar tidal radius. Due to that, one star is ejected away, thus being saved from tidal disruption, while the other one remains parked on a tightly bound orbit whose pericenter is, however, typically well above the stellar tidal radius. Orbit of the bound star may not further undergo Kozai-Lidov oscillations, as it has abruptly changed its orbital elements during the binary tidal disruption event. Hence, it may also be saved from being tidally disrupted.

Finally, let us recall that the process discussed in this work led to a substantial number of stars ejected from the stellar disc with velocities only marginally exceeding the escape velocity from the SMBH. These stars should be found in the Galactic bulge. Interestingly, several young stars at distances of the order of tens of parsecs from the SgrA ${ }^{\star}$ are observed. Their origin is unclear. Some of them may have been ejected from the young star clusters Arches and Quintuplet. However, it has been discussed by Habibi et al. (2014) that these two clusters could not be birth places of all of them. Let us close our discussion with a suggestion that also the young stellar disc may have contributed to this population of young stars.

\section{CONCLUSIONS}

We have investigated by means of direct $N$-body integrations the production of the HVSs and the S-stars from an eccentric stellar disc around the SMBH through tidal break-ups of binaries brought to the vicinity of the SMBH via the coplanar eccentric Kozai-Lidov mechanism induced by the stellar disc itself. In accord with the principle of the Occam's razor, this model of the origin of the HVSs and the S-stars ob- 
served in the Galactic halo and nucleus, respectively, relies on as small number of constituents as possible. At the same time, however, it assumes recurrent star formation episodes to occur in the Galactic center. The currently observed HVSs required at least $50 \mathrm{Myr}$ to travel from the Galactic center to the halo, i.e. they can not originate from the young stellar disc observed now in the Galactic center which is $\lesssim 7 \mathrm{Myr}$ old (e.g. Paumard et al. 2006; Lu et al. 2013).

Velocity distribution of the HVSs in our model as well as their total number strongly depends on the properties of the initial binary population. Unfortunately, this piece of information is unavailable for the case of the young stellar disc observed in the Galactic center, which otherwise, is considered to be a template configuration. Therefore, any strong statement on the viability of our model based on the observational data cannot be made at this moment. The most prominent 'smoking gun' of our hypothesis appears to be strong anisotropy of distribution of the HVSs on the sky which is a robust feature of the model and is in accord with the current observational data. Another characteristic feature of the HVSs in our model is a burst-like mode of their formation. This feature is neither confirmed, nor excluded for the currently observed HVSs (Brown et al. 2014). Future observations may bring stronger constraints in this point. Note however, that (i) in spite that our model predicts one dominant peak of ejection, still large fraction of HVSs are produced in wide time range and (ii) more sophisticated model including spherical cluster is likely to lower the dominant peak.

Hand in hand with production of the HVSs, our model of tidal break-ups of binary stars originating from an eccentric disc also leads to transportation of stars to orbits tightly bound to the SMBH within a few millions of years. These could be then observed as S-stars. Strong initial anisotropy of normal vectors of their orbital planes is very quickly (within another few millions of years) smeared out due to resonant relaxation within an embedding stellar cluster. Hence, unlike in the case of the HVSs, we do not observe any characteristic feature of the distribution functions of the orbital elements of the S-stars formed in our model which could serve as a strong test bed of its relevance. Finally, let us note that quite rapid formation of the population of the S-stars from an eccentric disc implies that, according to our model, some of the currently observed S-stars may have originated from the young stellar disc observed in the Galactic center nowadays. On the other hand, life-time of the observed S-stars allows them to be original companions of the HVSs currently observed in the Galactic halo, i.e. originating from some previous star formation episode. The two scenarios are not mutually exclusive. Actually, we estimate the number of S-stars originating from the currently observed young stellar disc in the Galactic center to be only a few when considering damping role of the spherical component of the nuclear star cluster.

Beside the properties of the HVSs and the S-stars, we also evaluated evolution of the parent stellar disc. We found that it evolves in a way similar to models without primordial binaries. In particular, the binaries do not contribute significantly to heating of the disc, i.e. the model of isolated disc is not able to reproduce spatial distribution of all the young stars in the Galactic center, substantial fraction of which are observed well above the plane of the young stellar disc (CWS). The hypothesis of common origin of all young stars observed in the GC from a single parent disc may be kept viable if we assume some other perturbing source(s) of gravity to be present (see, e.g., Subr et al. 2009; Haas et al. 2011a bor discussion of influence of an outer massive gaseous torus on evolution of the stellar disc).

\section{ACKNOWLEDGMENTS}

We thank the anonymous referee for helpful comments. L.S. acknowledges support from the Grant Agency of the Czech Republic via Grant No. GACR 14-10625S and from the National Science Foundation under Grant No. PHYS1066293 and the hospitality of the Aspen Center for Physics. J.H. was supported by Charles University project UNCE204020 .

\section{REFERENCES}

Aarseth, S. J. 2003, Gravitational N-Body Simulations (Cambridge: Cambridge Univ. Press)

Antonini, F., Faber, J., Gualandris, A., \& Merritt, D. 2010, ApJ, 713, 90

Antonini, F., Lombardi, \& J. C., Merritt, D. 2011, ApJ, 731, 128

Antonini, F., \& Merritt, D. 2013, ApJL, 763, L10

Bartko, H., Martins, F., Fritz, T. K., et al. 2009, ApJ, 697, 1741

Bartko, H., Martins, F., Trippe, S., et al. 2010, ApJ, 708, 834

Brown, W. R., Geller, M. J., \& Kenyon, S. J. 2014, ApJ, 787, 89

Chen, X., \& Amaro-Seoane, P. 2014, ApJL, 786, L14

Do, T., Lu, J. R., Ghez, A. M., et al. 2013, ApJ, 764, 154

Ford, E. B., Kozinsky, B., \& Rasio, F. A. 2000, ApJ, 535, 385

Ghez, A. M., Salim, S., Hornstein, S. D., et al. 2005, ApJ, 620, 744

Gillessen, S., Eisenhauer, F., Trippe, S., et al. 2009, ApJ, 692, 1075

Gould, A., \& Quillen, A. C. 2003, ApJ, 592, 935

Haas, J., Šubr, L., Kroupa, P. 2011a, MNRAS, 412, 1905

Haas, J., Šubr, L., Vokrouhlický, D. 2011b, MNRAS, 416, 1023

Haas, J., \& Subr, L. 2016, ApJ, 822, 25

Habibi, M., Stolte, A., \& Harfst, S. 2014, A\&A, 566, A6

Hopman, C., \& Alexander, T. 2006, ApJ, 645, 1152

Hills, J. G. 1988, Natur, 331, 687

Hills, J. G. 1991, AJ, 102, 704

Ivanov, P. B., Polnarev, A. G., \& Saha, P. 2005, MNRAS, 358, 1361

Karas, V., \& Šubr, L. 2007, A\&A, 470, 11

Katz, B., Dong, S., \& Malhotra, R. 2011, PhRvL, 107, 1101

Kenyon, S. J., Bromley, B. C., Geller, M. J., et al. 2008, ApJ, 680, 312

Kobulnicky, H. A., \& Fryer, C. L. 2007, ApJ, 670, 747

Komossa, S. 2015, JHEAp, 7, 148
Kozai, Y. 1962, AJ, 67, 591

Levin, Y., \& Beloborodov, A. M. 2003, ApJL, 590, L33

Li, G., Naoz, S., Kocsis, B., et al. 2014, ApJ, 785, 116

Lidov, M. L. 1962, Planet. Space Sci., 9, 719

Lithwick, Y., \& Naoz, S. 2011, ApJ, 742, 94

Löckmann, U., Baumgardt, H., \& Kroupa, P. 2008, ApJL, 683, L151

Lu, J. R., Do, T., Ghez, A. M., et al. 2013, ApJ, 764, 155

Madigan, A.-M., Levin, Y., \& Hopman, C. 2009, ApJL, 697, L44

Madigan, A.-M., Hopman, C., \& Levin, Y. 2011, ApJ, 738, 99

Mapelli, M., Hayfield, T., Mayer, L., et al. 2012, ApJ, 749, 168

Meyer, L., Ghez, A. M., Schödel, R., et al. 2012, Sci, 338, 84

Mikhaloff, D., \& Perets, H. B. 2016, arXiv:1504.05951

Paumard, T., Genzel, R., Martins, F., et al. 2006, ApJ, 643, 1011

Perets, H. B., Hopman, C., \& Alexander, T. 2007 ApJ, 656, 709

Perets, H. B., Gualandris, A., Kupi, G., et al. 2009, ApJ, 702, 884

Pfuhl, O., Alexander, T., Gillessen, S., et al. 2014, ApJ, 782, 101

Prodan, S., Antonini, F., \& Perets, H. B. 2015, ApJ, 799, 118

Rauch, K. P., \& Tremaine, S. 1996, NewA, 1, 149

Saxton, R. D., Read, A. M., Esquej, P., et al. 2012, A\&A, 541, A106

Stephan, A. P., Naoz, S., Ghez, A. M., et al. 2016, arXiv:1603.02709

Stewart, G. R., \& Ida, S. 2000, Icarus, 143, 28

Šubr, L., Karas, V., \& Haas, J. 2007, in proc. conf. IAU Symposium 238 "Black Holes from Stars to Galaxies - Across the Range of Masses", V. Karas and G. Matt eds., pp. 201-206

Šubr, L., Schovancová, J., Kroupa, P. 2009, A\&A, 496, 695

Šubr, L., \& Haas, J. 2014, ApJ, 786, 121

Vickers, J. J., Smith, M. C., \& Grebel, E. K. 2015, AJ, 150, 77 
Zhang, F., Lu, Y., Yu, Q. 2013, ApJ, 768, 153 